\title{
Formation of Cellular Flames and Increase in Flame Velocity Generated by Intrinsic Instability
}

\author{
By Satoshi KADOWAKI \\ Department of Mechanical Engineering, Nagaoka University of Technology, Nagaoka, Japan
}

(Received July 12th, 2001)

\begin{abstract}
The formation of cellular flames and the increase in flame velocity generated by intrinsic instability are studied by two-dimensional (2-D) and three-dimensional (3-D) unsteady calculations of reactive flows based on the compressible Navier-Stokes equation. We consider three basic types of phenomena to be responsible for the intrinsic instability of premixed flames, i.e., hydrodynamic, diffusive-thermal, and body-force instabilities. Cellular flames are generated by intrinsic instability, and thus the flame-surface area becomes larger and the flame velocity increases. The increment in flame velocity of 3-D flames is about twice that of 2-D flames, since the increment in flame-surface area of the former is about twice that of the latter. This relationship is due to the difference in the disposition of cells between 2-D and 3-D flames. When the Lewis number is lower than unity, i.e., when diffusive-thermal instability appears, the increment in flame velocity is larger than in the flame-surface area. This is because the increase in the local consumption rate of the unburned gas at a convex flame surface exceeds the decrease at a concave one. When the Lewis number is unity, i.e., when hydrodynamic and body-force instabilities are dominant, the flame velocity is nearly proportional to the flame-surface area, since the local consumption rate is nearly constant.
\end{abstract}

Key Words: Intrinsic Instability, Cellular Flame, Flame Velocity

\section{Introduction}

In premixed flames propagating at subsonic speed, e.g., hydrogen-air or hydrocarbon-air flames, intrinsic instability always appears. The basic types of phenomena responsible for the intrinsic instability of premixed flames are hydrodynamic, diffusive-thermal, and body-force instabilities. ${ }^{1-4)}$ Hydrodynamic instability is caused by the thermal expansion through the flame surface. ${ }^{5-7)}$ This type of instability is the most essential, since the combustion reaction is exothermic and all premixed flames are accompanied by thermal expansion. Thus we cannot study the instability of premixed flames without considering hydrodynamic instability. Diffusive-thermal instability, together with hydrodynamic instability, is important to intrinsic instability. This instability is caused by the preferential diffusion of mass versus heat and appears at the Lewis numbers of the deficient reactant lower than unity. ${ }^{8-10)}$ The typical example of diffusive-thermal instability is a cellular flame, which has been experimentally observed in lean (rich) gas mixtures with light (heavy) fuel. ${ }^{11-14)}$ Moreover, numerical investigations on cellular-flame formation at low Lewis numbers have been performed, based on either the diffusive-thermal model equation with a constant-density approximation ${ }^{15-19)}$ or on the compressible Navier-Stokes equation. ${ }^{20-23)}$ Bodyforce instability, which is caused by the difference in density between the upper and lower fluids, appears when premixed flames are propagated upward. ${ }^{24-27)}$ This is because the density of the upper fluid, i.e., the unburned gas, is higher than

(c) 2002 The Japan Society for Aeronautical and Space Sciences that of the lower fluid, i.e., the burned gas. The body force has a destabilizing (stabilizing) influence on upward (downward) propagating flames.

Because of intrinsic instability, an infinitesimal disturbance superimposed on a plane flame evolves, eventually forming a cellular flame. The flame surface becomes larger, so the flame velocity increases. Thus the increase in flame velocity is generated by intrinsic instability. When the Lewis number is unity, i.e., when hydrodynamic and body-force instabilities are dominant, the increase in flame velocity is due mainly to the increase in flame-surface area. ${ }^{28,29)}$ When the Lewis number is lower than unity, i.e., when diffusivethermal instability appears, the flame velocity of a cellular flame is not dependent only on the flame-surface area. Moreover, in turbulent premixed flames, for example, the increment in flame velocity is larger than that in the flamesurface area at Lewis numbers lower than unity ${ }^{30,31)}$ and at lean hydrogen-air mixtures. ${ }^{32}$ ) Thus we need to consider not only the flame-surface area, but also the Lewis-number effect in studying flame velocity.

The author has performed 2-D unsteady calculations of reactive flows, based on the compressible Navier-Stokes equation, to study the flame velocity of cellular flames at low Lewis numbers. ${ }^{33)}$ It has been reported that the increment in flame velocity is nearly equal to that in the flame-surface area at Lewis-number unity and that the former increment is larger than the latter one at Lewis numbers lower than unity. However, the 3-D reactive flows were not studied, even though 3-D flames are the types usually observed in experiments, and the difference in flame velocity between 2-D 
and 3-D cellular flames is significant. Moreover, the influence of hydrodynamic and body-force instabilities on flame velocity has not been discussed in detail.

In the present study, we calculate 2-D and 3-D unsteady reactive flows to study the formation of cellular flames and the increase in flame velocity generated by intrinsic instability. We obtained the relationship between flame velocity and flame-surface area to investigate the influence of intrinsic instability on flame velocity. Moreover, we present a comparison between 2-D and 3-D cellular flames.

\section{Governing Equations}

The 2-D and 3-D single-reactant flames are treated in the present study. It is assumed that the chemical reaction is a one-step irreversible exothermic reaction and that the reaction rate obeys Arrhenius's law. The unburned and burned gases have the same molecular weights and the same Lewis numbers, and they satisfy the ideal gas equation of state. The transport coefficients and specific heats are constant throughout the whole region. The Soret effect, Dufour effect, pressure gradient diffusion, bulk viscosity, and radiation are negligible. The body force acts only in the direction normal to the flame surface.

To consider hydrodynamic instability, which is the most essential to intrinsic instability, the compressible NavierStokes equation is employed. The viscous and body-force terms in the equation of energy conservation are disregarded, since their contribution is trivial in the present problem. Using Cartesian coordinates, we take the direction tangential to the flame surface as the $y z$-surface, with the gas velocity in the positive $x$-direction.

The flow variables are nondimensionalized by the characteristic length, the characteristic velocity, and the density of the unburned gas. The characteristic length is the preheat zone thickness, which is defined as the thermal diffusivity divided by the burning velocity, and the characteristic velocity is the burning velocity. The nondimensionalization of flow variables is described in detail in our former paper. ${ }^{29)}$ The nondimensionalized governing equations of 3-D unsteady reactive flows are written in the conservation form as

$$
\frac{\partial \boldsymbol{U}}{\partial t}+\frac{\partial \boldsymbol{F}}{\partial x}+\frac{\partial \boldsymbol{G}}{\partial y}+\frac{\partial \boldsymbol{H}}{\partial z}=\boldsymbol{S}
$$

where $\boldsymbol{U}, \boldsymbol{F}, \boldsymbol{G}, \boldsymbol{H}$, and $\boldsymbol{S}$ are vectors given by

$$
\boldsymbol{U}=\left(\begin{array}{c}
\rho \\
\rho u \\
\rho v \\
\rho w \\
e \\
\rho Y
\end{array}\right)
$$

$$
\boldsymbol{F}=\left(\begin{array}{l}
\rho u \\
\rho u^{2}+\frac{p}{\gamma M_{0}^{2}}-\operatorname{Pr}\left(\frac{4}{3} \frac{\partial u}{\partial x}-\frac{2}{3} \frac{\partial v}{\partial y}-\frac{2}{3} \frac{\partial w}{\partial z}\right) \\
\rho u v-\operatorname{Pr}\left(\frac{\partial v}{\partial x}+\frac{\partial u}{\partial y}\right) \\
\rho u w-\operatorname{Pr}\left(\frac{\partial w}{\partial x}+\frac{\partial u}{\partial z}\right) \\
(e+p) u-\frac{\gamma}{\gamma-1} \frac{\partial T}{\partial x} \\
\rho Y u-\frac{1}{L e} \frac{\partial Y}{\partial x}
\end{array}\right)
$$$$
\boldsymbol{G}=\left(\begin{array}{l}
\rho v \\
\rho u v-\operatorname{Pr}\left(\frac{\partial v}{\partial x}+\frac{\partial u}{\partial y}\right) \\
\rho v^{2}+\frac{p}{\gamma M_{0}^{2}}-\operatorname{Pr}\left(\frac{4}{3} \frac{\partial v}{\partial y}-\frac{2}{3} \frac{\partial u}{\partial x}-\frac{2}{3} \frac{\partial w}{\partial z}\right) \\
\rho v w-\operatorname{Pr}\left(\frac{\partial w}{\partial y}+\frac{\partial v}{\partial z}\right) \\
(e+p) v-\frac{\gamma}{\gamma-1} \frac{\partial T}{\partial y} \\
\rho Y v-\frac{1}{L e} \frac{\partial Y}{\partial y}
\end{array}\right)
$$$$
\boldsymbol{H}=\left(\begin{array}{l}
\rho w \\
\rho u w-\operatorname{Pr}\left(\frac{\partial w}{\partial x}+\frac{\partial u}{\partial z}\right) \\
\rho v w-\operatorname{Pr}\left(\frac{\partial w}{\partial y}+\frac{\partial v}{\partial z}\right) \\
\rho w^{2}+\frac{p}{\gamma M_{0}^{2}}-\operatorname{Pr}\left(\frac{4}{3} \frac{\partial w}{\partial z}-\frac{2}{3} \frac{\partial u}{\partial x}-\frac{2}{3} \frac{\partial v}{\partial y}\right) \\
(e+p) w-\frac{\gamma}{\gamma-1} \frac{\partial T}{\partial z} \\
\rho Y w-\frac{1}{L e} \frac{\partial Y}{\partial z}
\end{array}\right)
$$

$$
S=\left(\begin{array}{l}
0 \\
G \rho \\
0 \\
0 \\
Q B \rho Y \exp (-E / T) \\
-B \rho Y \exp (-E / T)
\end{array}\right)
$$

where $t$ is the time; $\rho$ is the density; $u, v$, and $w$ are $x$-, $y$-, and $z$-components of the velocity; $e$ is the stored energy; $Y$ is the mass fraction of the unburned gas; $p$ is the pressure; $T$ is the temperature; $\gamma$ is the ratio of specific heats; $M_{0}$ is the Mach number of the burning velocity; Pr is the Prandtl number; $L e$ is the Lewis number; $G$ is the acceleration; $Q$ is the heating value; $B$ is the frequency factor; and $E$ is the 


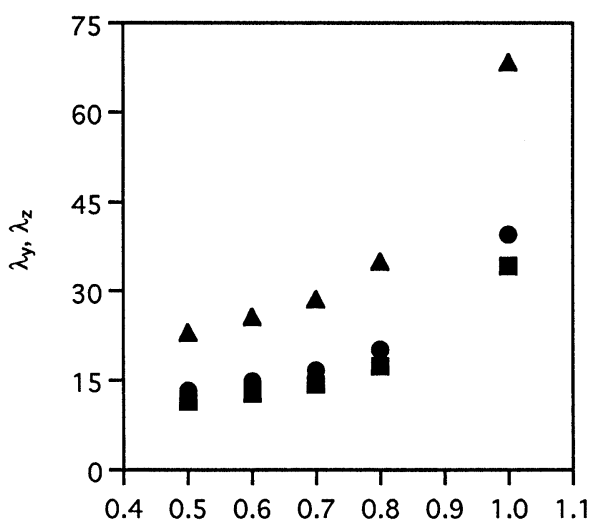

Le

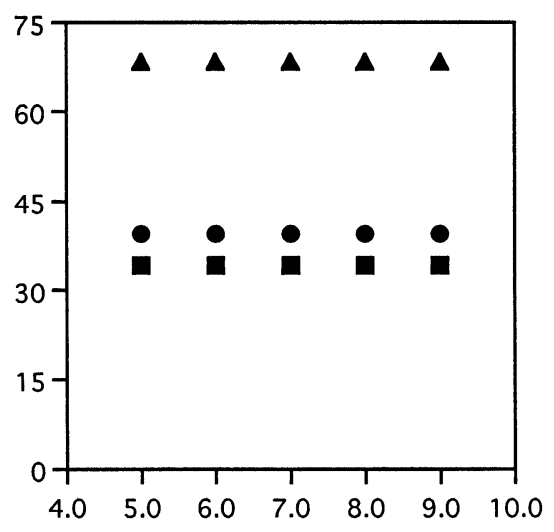

$T_{f}$

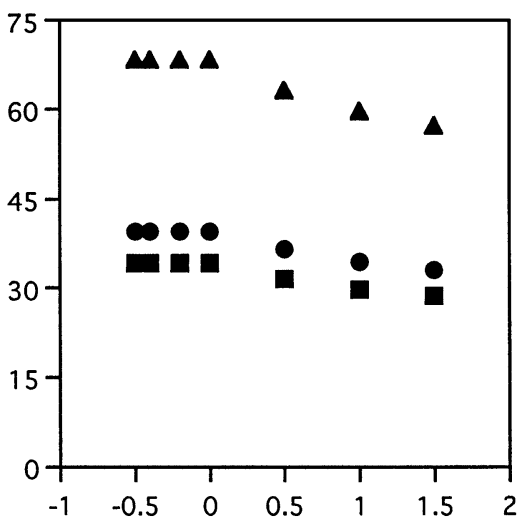

G

Fig. 1. Critical wavelengths at $L e=0.5-1.0, T_{\mathrm{f}}=5.0-9.0$, and $G=-0.5-1.5$; the closed square denotes the critical wavelength of 2-D flames, and the circle and triangle denote the critical wavelengths of 3-D flames in $y$ and $z$ directions, respectively.

activation energy. The equation of state is

$$
p=\rho T
$$

\section{Calculation Procedures}

We simulate the gas mixture, whose burning velocity is $0.83 \mathrm{~m} / \mathrm{s}$, at room temperature and atmospheric pressure. The burning velocity is sufficiently small compared with the sound velocity, i.e., $M_{0}=2.1 \times 10^{-3}$, so the nondimensionalized results hardly change, even though the burning velocity is changed. Nondimensional parameters are $\gamma=1.4$, $\operatorname{Pr}=1.0$, and $E=70$. The adiabatic flame temperature and Lewis number are taken $T_{\mathrm{f}}=5.0-9.0$ and $L e=0.5-1.0$, respectively. The acceleration is set to $G=-0.6-1.5$, where positive (negative) acceleration corresponds to upward (downward) propagating flames. The heating value and frequency factor are determined under the condition that the calculated adiabatic flame temperature and burning velocity of a plane flame are equal to the set values.

A disturbance periodic in $y$ and $z$ directions is superimposed on a stationary plane flame, and the evolution of a disturbed flame is calculated. The displacement of the flame surface in the $x$ direction because of the superimposed disturbance for 2-D flames is

$$
A_{0} \sin \left(2 \pi y / \lambda_{y}\right)
$$

For 3-D flames, a hexagonal disturbance is superimposed. ${ }^{34)}$

$$
A_{0}\left[\sin \left(2 \pi y / \lambda_{y}\right) \sin \left(2 \pi z / \lambda_{z}\right)-\cos \left(4 \pi z / \lambda_{z}\right) / 2\right]
$$

where $A_{0}$ is the initial amplitude, and $\lambda_{y}$ and $\lambda_{z}$ are the wavelengths in $y$ and $z$ directions, respectively. The relationship $\lambda_{z} / \lambda_{y}=\sqrt{3}$ is realized. The wavelength is set to the linearly most unstable wavelength, i.e., the critical wavelength (see Fig. 1), since the spacing between the cells of a cellular flame is equivalent to the critical wavelength.

The boundary conditions in the $x$ direction, except for the inlet velocity of the unburned gas, are provided by free-flow conditions. The inlet velocity is set equal to the burning ve- locity. The boundary conditions in $y$ and $z$ directions are provided by periodic conditions, since a sufficiently large flame-surface area is assumed. Thus the obtained results in the present calculations are valid for flames with a sufficiently large surface area.

As for numerical scheme, the explicit MacCormack scheme, which has second-order accuracy in both time and space, is adopted. The computational domain is 160 times the preheat zone thickness in the $x$ direction, and one wavelength of a disturbance in the $y$ and $z$ directions. The variably spaced grid is used in the $x$ direction, where the minimum grid size is a fifth of the preheat zone thickness. In $y$ and $z$ directions, the uniformly spaced grid is used. The computational domain is resolved by a $341 \times 65$ grid for 2 D flames and by a $261 \times 31 \times 53$ grid for 3 -D flames. The used grid is fine enough to calculate the present problem. When the grid size is halved, no significant difference is observed. The time-step interval is set to satisfy the CourantFriedrichs-Lewy condition.

\section{Results and Discussion}

We first studied the influence of diffusive-thermal instability $(L e<1)$ on the formation of cellular flames and the increase in flame velocity. The shapes of 2-D flames at $L e=0.8$ and 1.0 are illustrated in Fig. 2. The flame position is defined as the site where the reaction rate takes a maximum value. The unburned gas flows in from the left at the burning velocity, and the burned gas flows out to the right. The disturbance superimposed on a stationary plane flame grows with time as a result of intrinsic instability. When the amplitude grows to a certain degree, the disturbance is not evolved and the flame shape becomes cellular. The cell depths at $L e=0.8$ and 1.0 are 5.4 and 10.7, respectively. The flame-surface area of a cellular flame, which is normalized by that of a plane flame, at $L e=0.8$ is slightly smaller than that at $L e=1.0$. After cell formation, the cellular flame moves upstream, maintaining a cellular shape. This indicates that the flame velocity of a cellular flame is larger than that of 


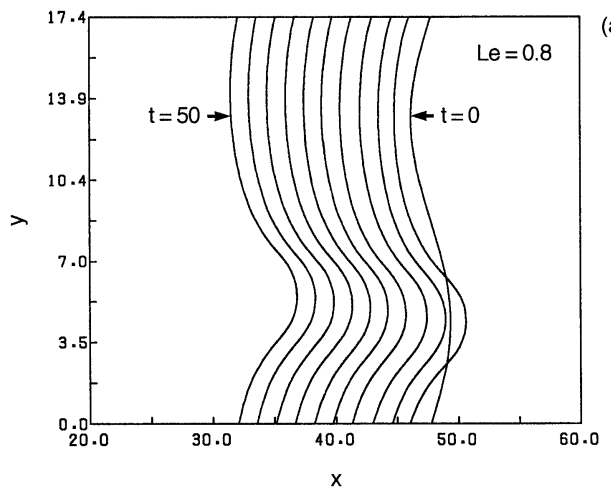

(a)

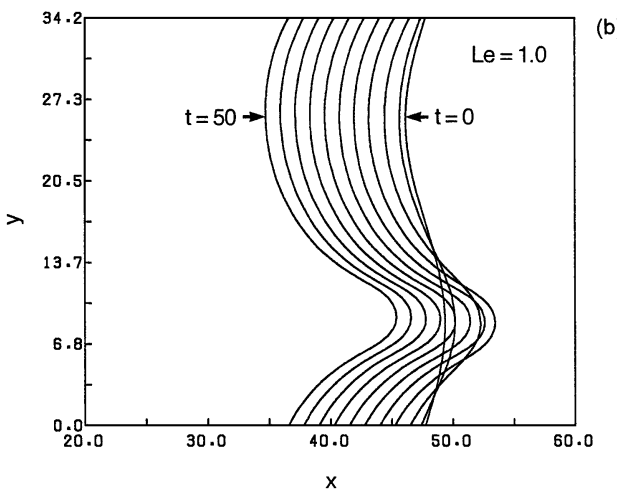

(b)

Fig. 2. Shapes of 2-D flames at $T_{\mathrm{f}}=7.0, G=0.0$, and $A_{0}=1.6$ $(t=0,5,10, \cdots, 50)$. (a) $L e=0.8$ and $\lambda_{y}=17.4$; (b) $L e=1.0$ and $\lambda_{y}=34.2$.

a plane flame, which is due to the increase in flame-surface area. The moving rate of a cellular flame is equivalent to the increment in flame velocity, since the inlet velocity is equal to the flame velocity of a plane flame. The increments in flame velocity at $L e=0.8$ and 1.0 are 0.30 and 0.24 , respectively. Thus the flame velocities are 1.30 and 1.24 , respectively. Although the cell depth and flame-surface area of the $L e=0.8$ flame are smaller than those of the $L e=1.0$ flame, the flame velocity of the former flame is larger than that of the latter flame, since diffusive-thermal instability appears at Le $<1$.

The shapes of 3-D cellular flames at $L e=0.8$ and 1.0 are illustrated in Fig. 3 . The schematic domain is $3 \lambda_{y} \times 2 \lambda_{z}$. The unburned gas flows in from the bottom, and the burned gas flows out to the top. The superimposed disturbance evolves, and a hexagonal cellular flame is formed $(t=20)$. The cell depths at $L e=0.8$ and 1.0 are 9.7 and 20.4, respectively. These values are much larger than those of 2-D flames. The distributions of cells at the $y z$-plane are illustrated in Fig. 4. Well-regulated hexagonal cell distribution is observed, and the spacing between cells is equal to $\lambda_{y}$. The hexagonal shape of 3-D cellular flames is in qualitative agreement with the experimental results. ${ }^{13)}$ After cell formation, the cellular flames move upstream, just as the 2-D cellular flames did. The increments in the flame velocity of 3-D flames at $L e=0.8$ and 1.0 are 0.71 and 0.59 , respectively.

Changing the Lewis number, we obtained the cell depth
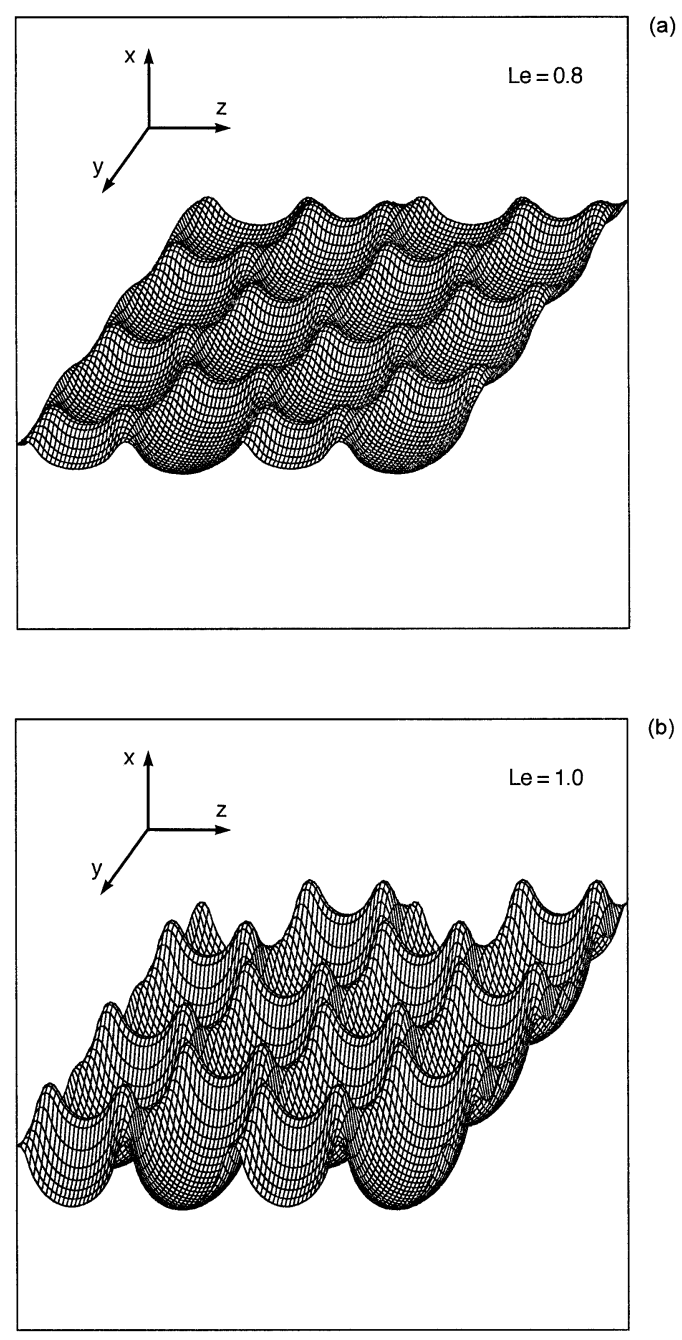

Fig. 3. Shapes of 3-D flames at $T_{\mathrm{f}}=7.0$ and $G=0.0(t=20)$ : (a) $L e=0.8, \lambda_{y}=20.1, \lambda_{z}=34.9$, and $A_{0}=0.8$; (b) $L e=1.0, \lambda_{y}=39.5$, $\lambda_{z}=68.4$, and $A_{0}=1.6$.

$D_{\text {cell }}$ and the ratio of cell depth to cell size $D_{\text {cell }} / \lambda_{y}$ of 2-D and 3-D flames (see Fig. 5). The obtained values are considered to be convergent values, since the variation of these values at the last stage of calculations is sufficiently small. The cell depth has a minimum value at $L e=0.7$ and increases by decreasing $L e$. This is due to a decrease in cell size with a decrease in Lewis number and to an increase in instability level. The ratio of cell depth to the cell size of the $L e=0.5$ flame is larger than that of the $L e=1.0$ flame, since diffusivethermal instability is stronger. Thus the flame-surface area of the former flame is larger than that of the latter flame. On the other hand, the ratio of the $L e=0.8$ flame is smaller than that of the $L e=1.0$ flame, even though the diffusivethermal instability appears at $L e=0.8$. This is because the flame has a finite thickness and the curvature at a concave flame surface with respect to the unburned gas is finite.

Figure 6 shows the dependence of flame velocity $S_{\text {cf }}$ and flame-surface area $L_{\mathrm{cf}}$ on the Lewis number. As the Lewis number decreases, the flame velocity increases. The increment in flame velocity of 3-D flames is about twice that of 

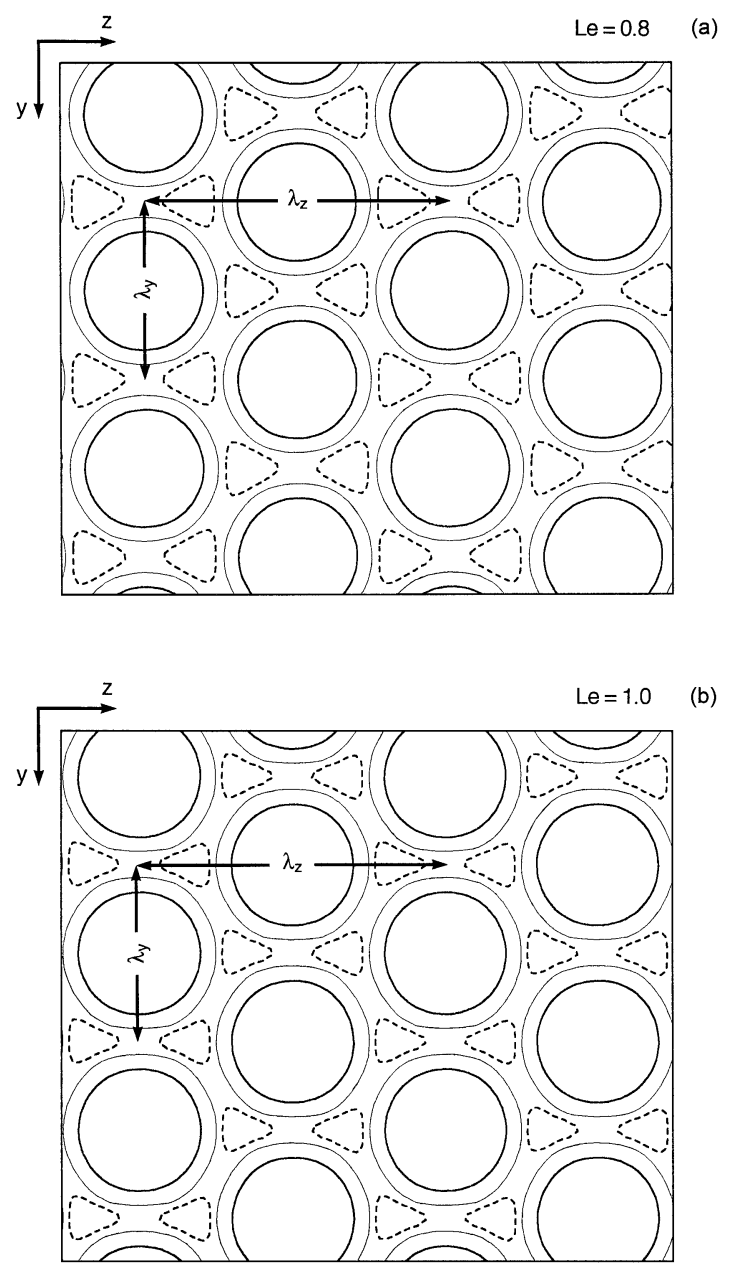

Fig. 4. Distributions of cells at the $y z$-plane at $T_{\mathrm{f}}=7.0$ and $G=0.0$ ( $t=20$ ): (a) $L e=0.8, \lambda_{y}=20.1, \lambda_{z}=34.9$, and $A_{0}=0.8$; (b) $L e=1.0, \lambda_{y}=39.5, \lambda_{z}=68.4$, and $A_{0}=1.6$.

2-D flames. This is due to the difference in the disposition of cells. When the Lewis number is unity, the nondimensionalized flame velocity is nearly equal to the normalized flame-surface area. This means that the flame velocity is nearly proportional to the flame-surface area. When the Lewis number is lower than unity, however, the increment in flame velocity is larger than that in flame-surface area. This difference becomes conspicuous as the Lewis number becomes lower.

The relationship between flame velocity and flamesurface area at $L e<1$ is essentially different from the relationship at $L e=1$. When $L e<1$, the reaction rate increases (decreases) at a convex (concave) flame surface with respect to the unburned gas. This is because the flame temperature becomes higher (lower) at a convex (concave) flame surface because of the preferential diffusion of mass versus heat. As the Lewis number becomes lower, the dependence of local reaction rate on flame shape becomes conspicuous. When $L e=1$, however, the reaction rate has a constant value at the flame surface, and its value is nearly equal to that of a plane flame.

The local reaction rate depends on the flame shape at
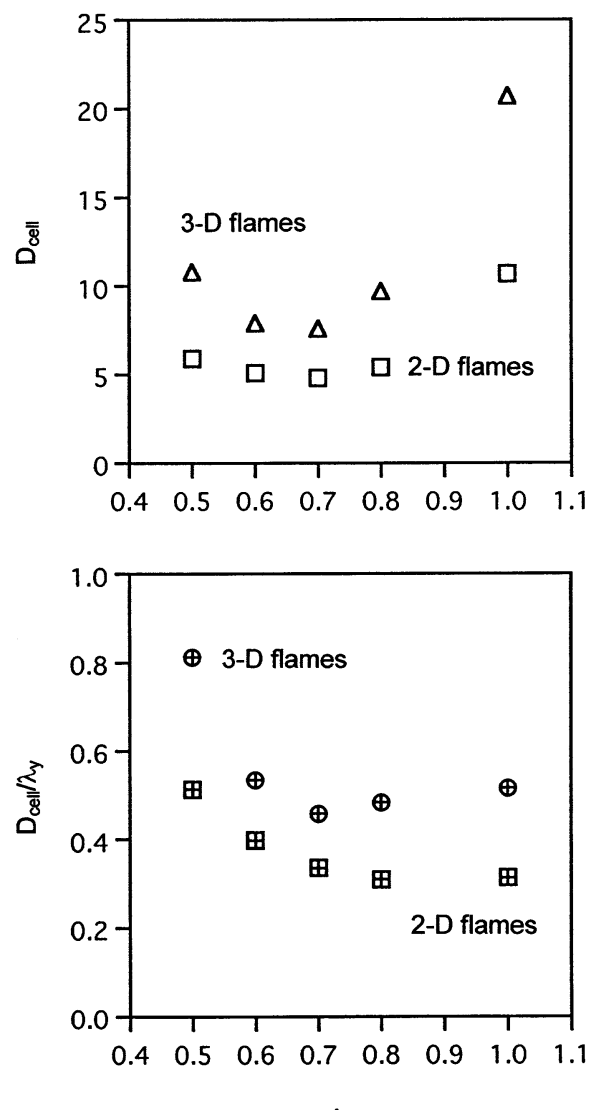

Le

Fig. 5. Cell depth and the ratio of cell depth to cell size, depending on the Lewis number, at $L e=0.5-1.0, T_{\mathrm{f}}=7.0$, and $G=0.0$.

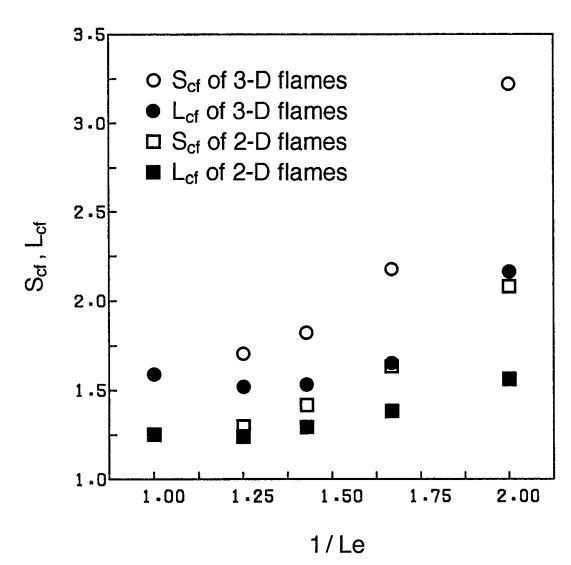

Fig. 6. Flame velocity and flame-surface area of 2-D and 3-D cellular flames, depending on the Lewis number, at $L e=0.5-1.0, T_{\mathrm{f}}=7.0$, and $G=0.0$.

$L e<1$, so the local consumption rate of the unburned gas also depends on it. In the present problem, the inlet flow excludes disturbances, and then the local consumption rate depends mainly on the curvature of the flame surface. This is because the flame curvature has the most important influence on the flame stretch by which the local consumption rate is governed. ${ }^{3)}$ Figure 7 shows the relationship between the local consumption rate of the unburned gas $s^{*}$ and the flame curvature $\kappa$. The local consumption rate was obtained by in- 

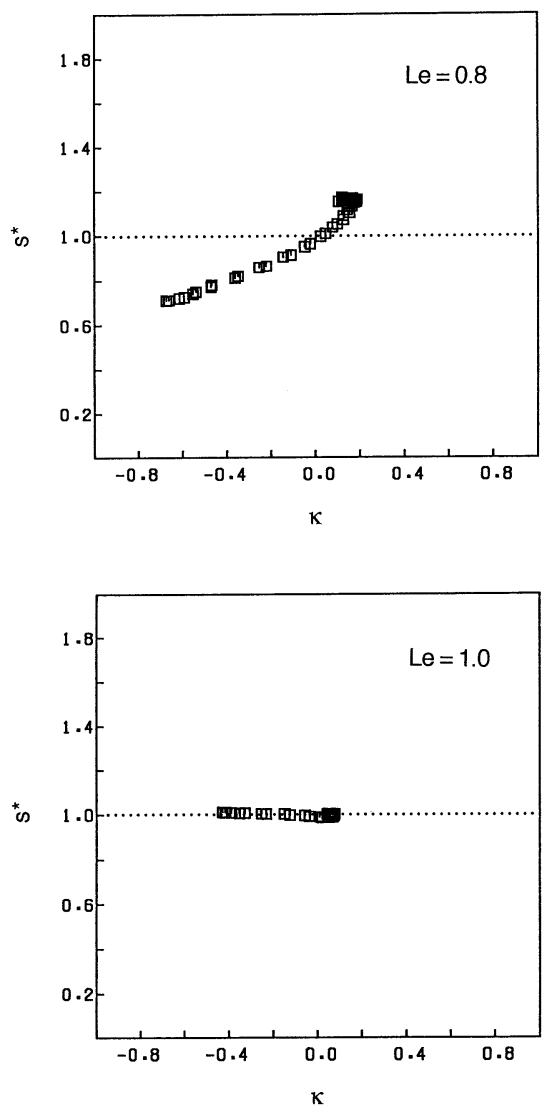

Fig. 7. Relationship between the local consumption rate of the unburned gas and the flame curvature at $L e=0.8$ and $1.0(t=25)$.

tegrating the reaction rate in the direction normal to the flame surface, and its value is normalized by that of a plane flame. The flame curvature is defined as positive at a convex flame surface with respect to the unburned gas. When $L e=0.8$ $(<1)$, the local consumption rate increases (decreases) with a positive (negative) flame curvature. Integrating the curvature along the flame surface from $y=0$ to $y=\lambda_{y}$, the integral becomes zero, since periodic conditions are used in the $y$-direction. If the dependence of local consumption rate on flame curvature were linear, the increase in consumption rate would equal the decrease. In this case $(L e<1)$, however, the dependence is nonlinear; i.e., the larger the flame curvature, the greater the rate of increment in local consumption rate. Thus the increase in consumption rate at a convex flame surface exceeds the decrease at a concave one. Therefore the flame-velocity increment is greater than the surfacearea increment. When $L e=1.0$, on the other hand, the local consumption rate is independent of the flame curvature and is nearly constant. Thus the flame velocity is nearly proportional to the flame-surface area. Strictly speaking, the local consumption rate depends on the flame curvature, even at $L e=1$; however, the dependence on the flame curvature is trivial in the present problem, since the spacing between cells is much larger than the preheat zone thickness and the flame curvature is comparatively small $(\kappa<1)$.

The dependence of local consumption rate on the flame curvature at $L e<1$ is explained as follows: At a convex (concave) flame surface, the temperature becomes higher (lower), and the reaction rate increases (decreases). The reaction rate obeys Arrhenius's law, so it grows exponentially with temperature. As the flame curvature becomes larger, the flame temperature becomes higher and the rate of increment in local consumption rate increases. Therefore, the increase in consumption rate at a convex flame surface exceeds the decrease at a concave one; thus the increment in flame velocity is larger than that in a flame-surface area.

The mean of local flame velocity is defined as the ratio of the flame velocity to the flame-surface area. This value is unity at $L e=1$ and increases as the Lewis number decreases, since diffusive-thermal instability becomes stronger. The mean of local flame velocity of 3-D flames is greater than that of 2-D flames. The reason for this is that the flame curvature at a convex flame surface of 3-D flames is larger. Compared with the 2-D flames, the influence of diffusivethermal instability on the flame velocity is prominent in 3-D flames.

Second, we studied the influence of hydrodynamic instability ( $L e=1, G=0$ ) on the structure of cellular flames. Changing the adiabatic flame temperature, we obtained the cell depth and the ratio of cell depth to cell size of 2-D and 3D flames (see Fig. 8). As the adiabatic flame temperature becomes higher, the cell depth increases, since hydrodynamic instability caused by thermal expansion becomes stronger. The dependence of the ratio $D_{\text {cell }} / \lambda_{y}$ on the temperature is the same as that of the cell depth, since the cell size is constant.

The flame velocity at $T_{\mathrm{f}}=5.0-9.0$ is shown in Fig. 9 . With an increase in adiabatic flame temperature, the flamesurface area becomes larger and the flame velocity increases. This is due to the increase in instability level. The Lewis number is set to unity; i.e., diffusive-thermal instability disappears, so the flame velocity is nearly proportional to the flame-surface area. ${ }^{28)}$ The increment in flame velocity of the 3 -D flames is about twice that of the 2-D flames, which is the same as the results shown in Fig. 6 .

Finally, we studied the influence of body-force instability. The adiabatic flame temperature and Lewis number were set at 7.0 and 1.0, respectively, since we gave attention to body-force instability. When the acceleration is positive (negative), the body force has a destabilizing (stabilizing) influence. Changing the acceleration, the cellular structure of upward or downward propagating flames was calculated. The cell depth and the ratio of cell depth to cell size at $G=-0.6-1.5$ are shown in Fig. 10 . When $G>0$, the flame velocity is increased as the acceleration becomes larger, i.e., the instability level becomes higher. When $G<0$, the flame velocity is smaller than that at $G=0$, and no cellular flame is formed at $G \leq-0.6$. This is because the body force, which has a stabilizing influence at $G<0$, prevents hydrodynamic instability from destabilizing the flame surface.

A cellular flame is formed at $G>-0.6$ because of intrinsic instability. The flame velocity is nearly proportional 

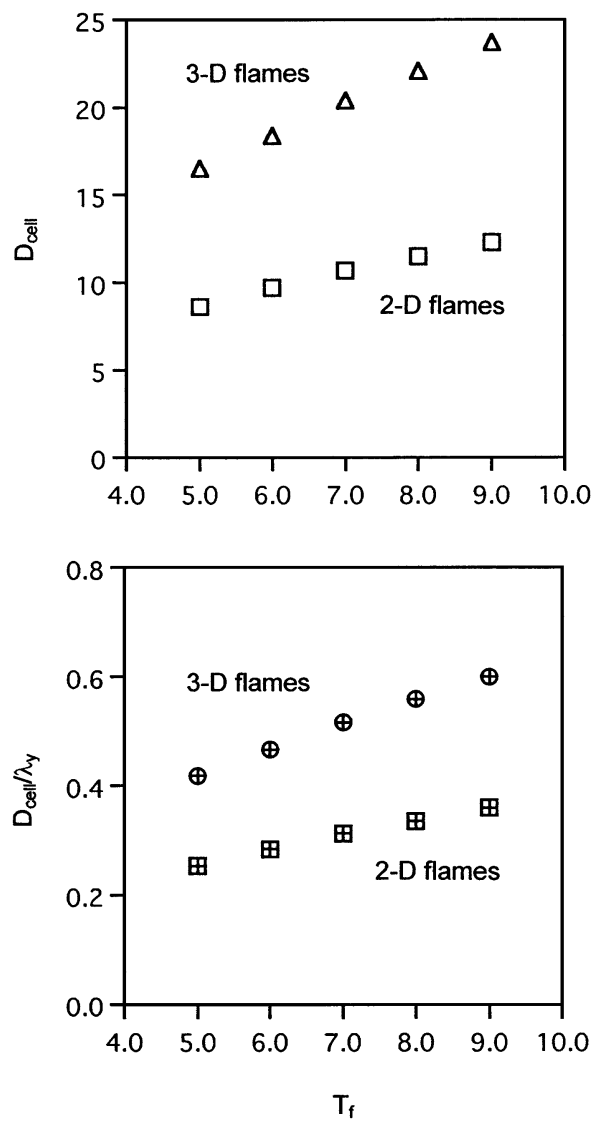

Fig. 8. Cell depth and the ratio of cell depth to cell size, depending on the adiabatic flame temperature, at $L e=1.0, T_{\mathrm{f}}=5.0-9.0$, and $G=0.0$.

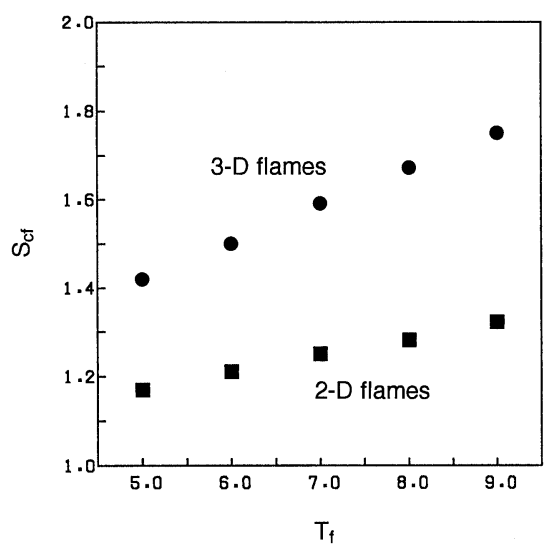

Fig. 9. Flame velocity of 2-D and 3-D cellular flames, depending on the adiabatic flame temperature, at $L e=1.0, T_{\mathrm{f}}=5.0-9.0$, and $G=0.0$.

to the flame-surface area, ${ }^{29)}$ even though the acceleration is changed (see Fig. 11), because the local consumption rate is nearly constant. Thus the body force has no influence on the mean of local flame velocity at $L e=1$. The increment in flame velocity of 3-D flames is about twice that of 2-D flames, as shown in Figs. 6 and 9. This relationship is not due to the factors responsible for intrinsic instability, i.e., the thermal expansion, preferential diffusion, and body force, but to the difference in the disposition of cells between 2-D and 3-D flames.
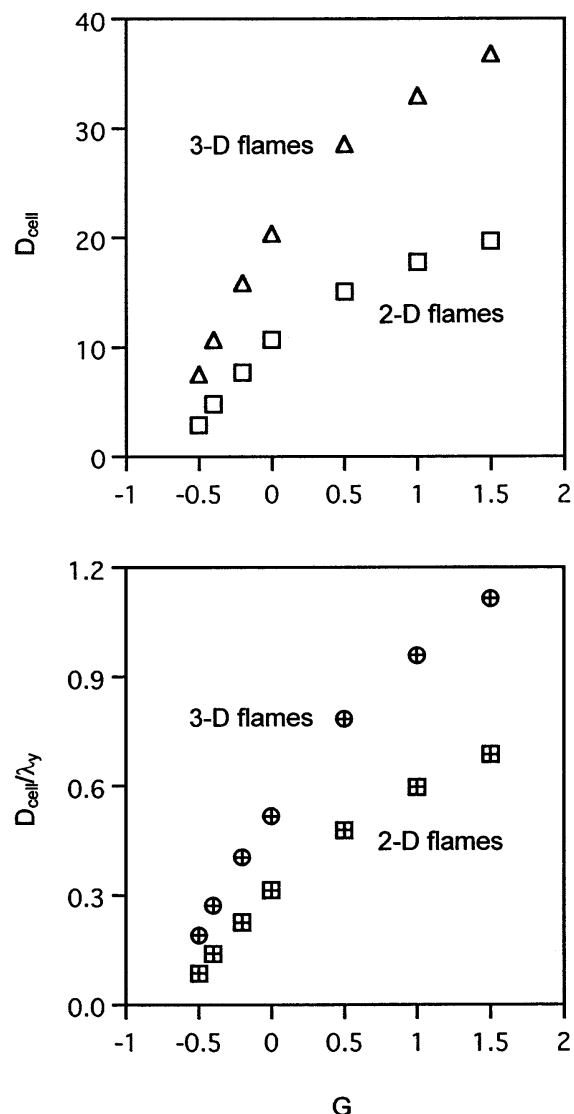

Fig. 10. Cell depth and the ratio of cell depth to cell size, depending on the acceleration, at $L e=1.0, T_{\mathrm{f}}=7.0$, and $G=-0.6-1.5$.

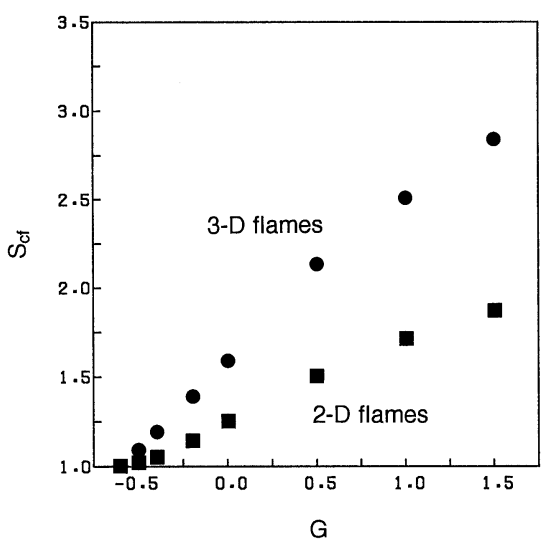

Fig. 11. Flame velocity of 2-D and 3-D cellular flames, depending on the acceleration, at $L e=1.0, T_{\mathrm{f}}=7.0$, and $G=-0.6-1.5$.

\section{Concluding Remarks}

We have performed 2-D and 3-D unsteady calculations of reactive flows, based on the compressible Navier-Stokes equation, to study the formation of cellular flames and increase in flame velocity generated by intrinsic instability. As intrinsic instability becomes stronger, the flame-surface area becomes larger and flame velocity increases. The increment in flame velocity of 3-D flames is about twice that of 2-D flames, which is due to the difference in the disposition of 
cells between 2-D and 3-D flames. When $L e<1$, the increment in flame velocity is larger than in the flame-surface area, since the increase in the local consumption rate of the unburned gas at a convex flame surface exceeds the decrease at a concave one. When $L e=1$, however, the flame velocity is nearly proportional to the flame-surface area, since the local consumption rate is nearly constant.

\section{References}

1) Clavin, P.: Dynamic Behavior of Premixed Flame Fronts in Laminar and Turbulent Flows, Prog. Energy Combust. Sci., 11 (1985), pp. 159.

2) Williams, F. A.: Combustion Theory, 2nd ed., Addison-Wesley, Reading, Massachusetts, 1985, pp. 349-365.

3) Law, C. K.: Dynamics of Stretched Flames, Proc. Combust. Inst., 22 (1988), pp. 1381-1402.

4) Sivashinsky, G. I.: On the Intrinsic Dynamics of Premixed Flames, Philos. Trans. Roy. Soc. A, 332 (1990), pp. 135-148.

5) Darrieus, G.: Propagation d'un Front de Flame, Unpublished Works Presented at La Technique Moderne, 1938

6) Landau, L. D.: On The Theory of Slow Combustion, Acta Physicochim, URSS, 19 (1944), pp. 77-85.

7) Kadowaki, S.: Instability of a Deflagration Wave Propagating with Finite Mach Number, Phys. Fluid, 7 (1995), pp. 220-222.

8) Barenblatt, G. I., Zeldovich, Y. B. and Istratov, A. G.: On DiffusiveThermal Stability of a Laminar Flame, J. Appl. Mech. Tech. Phys., 4 (1962), pp. 21-26.

9) Sivashinsky, G. I.: Diffusional-Thermal Theory of Cellular Flames, Combust. Sci. Technol., 15 (1977), pp. 137-146.

10) Joulin, G. and Mitani, T.: Linear Stability Analysis of Two-Reactant Flames, Combust. Flame, 40 (1981), pp. 235-246.

11) Markstein, G. H.: Experimental and Theoretical Studies of FlameFront Stability, J. Aeronaut. Sci., 18 (1951), pp. 199-209.

12) Hertzberg, M.: Selective Diffusional Demixing: Occurrence and Size of Cellular Flames, Prog. Energy. Combust. Sci., 15 (1989), pp. $203-$ 239.

13) Searby, G. and Quinard, J.: Direct and Indirect Measurements of Markstein Numbers of Premixed Flames, Combust. Flame, 82 (1990), pp. 298-311.

14) Gorman, M., el-Hamdi, M. and Robbins, K. A.: Experimental Observation of Ordered States of Cellular Flames, Combust. Sci. Technol., 98 (1994), pp. 37-45.

15) Hyman, J. M. and Nicolaenko, B.: The Kuramoto-Sivashinsky Equation: A Bridge between PDE's and Dynamical Systems, Physica D, 18 (1986), pp. 113-126.

16) Joulin, G. and Cambray, P.: On a Tentative, Approximate Evolution Equation for Markedly Wrinkled Premixed Flames, Combust. Sci.
Technol., 81 (1992), pp. 243-256.

17) Denet, B. and Haldenwang, P.: Numerical Study of Thermal-Diffusive Instability of Premixed Flames, Combust. Sci. Technol., 86 (1992), pp. 199-221.

18) Bayliss, A., Matkowsky, B. J. and Riecke, H.: Structure and Dynamics of Modulated Traveling Waves in Cellular Flames, Physica D, 74 (1994), pp. 1-23.

19) Daumont, I., Kassner, K., Misbah, C. and Valance, A.: Cellular SelfPropulsion of Two-Dimensional Dissipative Structures and SpatialPeriod Tripling Hopf Bifurcation, Phys. Rev. E, 55 (1997), pp. 6902 6906.

20) Patnaik, G. and Kailasanath, K.: Numerical Simulations of BurnerStabilized Hydrogen-air Flames in Microgravity, Combust. Flame, 99 (1994), pp. 247-253.

21) Denet, B. and Haldenwang, P.: A Numerical Study of Premixed Flames Darrieus-Landau Instability, Combust. Sci. Technol., 104 (1995), pp. 143-167.

22) Bychkov, V. V., Golberg, S. M., Liberman, M. A., Kleev, A. I. and Eriksson, L. E.: Numerical Simulation of Curved Flames in Cylindrical Tubes, Combust. Sci. Technol., 129 (1997), pp. 217-242.

23) Kadowaki, S.: Body-force Effect on the Lateral Movement of Cellular Flames at Low Lewis Numbers, Phys. Rev. E, 63 (2001), 026303.

24) Markstein, G. H.: Nonsteady Flame Propagation, Pergamon, Oxford, U. K. (1964), pp. 49-58.

25) Pelce, P. and Clavin, P.: Influence of Hydrodynamics and Diffusion Upon the Stability Limits of Laminar Premixed Flames, J. Fluid Mech., 124 (1982), pp. 219-237.

26) Dunsky, C. M.: Microgravity Observations of Premixed Laminar Flame Dynamics, Proc. Combust. Inst., 24 (1992), pp. 177-187.

27) Durox, D.: Effects of Gravity on Polyhedral Flames, Proc. Combust. Inst., 24 (1992), pp. 197-204.

28) Kadowaki, S.: The Influence of Hydrodynamic Instability on the Structure of Cellular Flames, Phys. Fluids, 11 (1999), pp. 3426-3433.

29) Kadowaki, S.: The Body-force Effect on the Cell Formation of Premixed Flames, Combust. Flame, 124 (2001), pp. 409-421.

30) Haworth, D. C. and Poinsot, T. J.: Numerical Simulations of Lewis Number Effects in Turbulent Premixed Flames, J. Fluid Mech., 244 (1992), pp. 405-436.

31) Hasegawa, T., Kuchita, M. and Yamaguchi, S.: Interaction of Premixed Flames with High-intensity Two-dimensional Turbulence, Nensho no Kagaku to Gijutsu (Japanese language companion publication to Combust. Sci. Technol.), 2 (1994), pp. 77-87.

32) Tanahashi, M., Miyauchi, T. and Nada, Y.: Turbulence-flame Interaction in $\mathrm{H}_{2}$-air Turbulent Premixed Flames, Nensho no Kagaku to Gijutsu (Japanese language companion publication to Combust. Sci. Technol.), 6-suppl. (1998), pp. 13-22.

33) Kadowaki, S.: Flame Velocity of Cellular Flames at Low Lewis Numbers, Combust. Sci. Technol., 162 (2001), pp. 223-234.

34) Christopherson, D. G.: Note on the Vibration of Membranes, Quarterly J. Math., 11 (1940), pp. 63-65. 